\title{
Negotiating Transmission Line Congestion Problems by Optimized Load Shedding Strategy
}

\author{
N.Ashokkumar \\ EEE Department, SCSVMV University, \\ Enathur, Kanchipuram- 631561.
}

\author{
M.Rathinakumar, $\mathrm{PhD}$ \\ EEE Department, SCSVMV University, \\ Enathur, Kanchipuram- 631561.
}

\begin{abstract}
In the present scenario, where there is steep rise in load demand and also where the electrical utility providers are unable to negotiate the demand rise by increasing the generation facilities, has resulted in transmission line congestion. Even though there are some solutions from FACTS controllers for tackling congestion in lines but it is not enough to tackle the problem. So as a last resort load shedding is the option available and followed. In this paper fuzzy expert system based approach is used to minimise the amount of load to be shed during the crisis. This has shown some improvement compared to conventional load shedding procedure.
\end{abstract}

\section{General Terms}

This paper gives some solution for transmission congestion; load shedding comes as a last resort for managing transmission congestion issues.

\section{Keywords}

Expert system approaches, fuzzy expert system, load shedding procedure, transmission line congestion.

\section{INTRODUCTION}

Transmission line congestion has many reasons associated with it. The rise in demand is very steep for the reasons of rise in electrical utility appliances which is now the order of the day. So the developing countries where there is free market cannot ration the usage of electrical appliances. This has steeply increased the demand. Increasing the generation facilities has its own challenges. So to manage the situation the transmission line facilities are operating to its close limits. This has stressed the power system infrastructure resulting in transmission line congestion. Congestion can be viewed as if the power exchanges were not controlled, some lines located on particular paths may become overloaded, and this phenomenon is called congestion. In a competitive electricity market, congestion occurs when the transmission network is unable to accommodate all of the desired transactions due to violation of system operating limits [1]. Congestion management can be defined as the quick operations taken by the technical persons to relieve the problem. One such approach is intelligent load shedding. Different load shedding schemes have been used in industrial power systems. Industries are more likely to use under-frequency load shedding schemes and this is based on frequency decay. Considering a single scheme for only one situation is not enough to resolve both frequency and voltage problems simultaneously especially for a large distribution network. Load shedding schemes can be generally less accurate and inefficient to operate. They can shed excessive load which is undesirable as it causes inconvenience to the customers, decreased revenues. [2]. Generally, a load shedding scheme integrates a stage-wise approach [3-4].The total amount of load to be shed depends on frequency deviation. If the frequency decreases to the first reference point then certain amount of load is shed to bring back to the reference point .This continues until system frequency is restored. In this process of load shedding care must be taken in such a way that excessive shedding is also avoided. If excessive load shedding in the system is done, there will be sudden change in the system voltage profile that causes transients in the system.

\section{LOAD SHEDDING FUNDAMENTALS}

In this work the data's from a substation are taken and with these data's an idea for optimum load shedding is arrived using fuzzy expert systems. The below are the methods followed by utility providers for load shedding.

\subsection{Breaker Interlock Load Shedding}

This is the simplest method of carrying out load shedding. For this scheme, the circuit breaker interdependencies are arranged to operate based on hardwired trip signals from an intertie circuit breaker or a generator trip. This method is often used when the speed of the load shedding is critical. Even though, the execution of this scheme is fast, breaker interlock load shedding possesses number of inherent drawbacks.

\subsection{Under-Frequency Relay Load Shedding}

Guidelines for setting up a frequency load shedding are common to both large and small systems. The design methodology considers fixed load reduction at fixed system frequency levels. Upon reaching the frequency set point and expiration of pre-specified time delay, the frequency relay trips one or more load breakers. This cycle is repeated until the system frequency is recovered, e.g., 10\% load reduction for every $0.5 \%$ frequency reduction. Since this method of load shedding can be totally independent of the system dynamics, total loss of the system is an assumed possibility.

\subsection{Programmable Logic Controller-Based Load Shedding}

With a Programmable Logic Controller (PLC) scheme, load shedding is initiated based on the total load versus the number of generators online and/or detection of under-frequency conditions. Each substation PLC is programmed to initiate a trip signal to the appropriate feeder breakers to shed a preset sequence of loads. This static sequence is continued until the frequency returns to a normal, stable level. A PLC-based load shedding scheme offers many advantages such as the use of a distributed network via the power management system, as well as an automated means of load relief. However, in such applications monitoring of the power system is limited to a portion of the network with the acquisition of scattered data. This drawback is further compounded by the implementation of pre-defined load priority tables at the PLC level that are executed sequentially to curtail blocks of load regardless of the dynamic changes in the system loading, generation, or operating configuration. The system-wide operating condition is often missing from the decision-making process resulting in 
insufficient or excessive load shedding. In addition, response time (time between the detection of the need for load shedding and action by the circuit breakers) during transient disturbances is often too long requiring even more load to be dropped.

\section{CLASSICAL ANALYSIS ON LOAD SHEDDING BY EARLIER LITERATURES}

Load shedding can be done by under frequency method which we generally mention it as UFLS strategy, also under voltage method generally mentioned UVLS. But in coordinated strategy both UFLS and UVLS is taken into effect which has improved results [2]. There are also strong support from literature in determining the apt location for load shedding and the amount to be shed for this non-linear optimization techniques are used [5].Similarly load shedding is determined by identifying the weakest node in the network using a voltage collapse proximity indicator (VCPI). The magnitude of the VCPI is determined by estimating the ratio of the impedance of the load to the Thevenin impedance looking into the network at a given voltage.[6].There are also approaches with specific interest on distribution side load shedding based on distribution reliabity index[7].

\section{COMPARISON BETWEEN THE METHODOLOGIES}

Even though there are number of methods available as discussed above, and all have their respective merits. This paper can be welcomed for the reason that here the data's are taken from practical case. That is data's are from substation and these data's are applied over fuzzy logic to get the desired benefit. In nutshell a visual scaling is done by fuzzy tool box and there is significant improvement over results. If this fuzzy logic control is implemented at substations, then there is smooth shedding of load and the value is greatly reduced compared to conventional methodology.

\section{PROBLEM FORMULATION PROCEDURE}

The following is the procedure we adopted for problem formulation.

1. Collection of data's from the sub-station.

2. Tabulation of the values of Voltage, Reactive power and Real power.

3. Find out the value of maximum permissible value of voltage, reactive power and real power.

4. Calculate $\mathrm{dV} / \mathrm{dQ}$ (voltage maximum -bus voltage/reactive power maximum-bus reactive power)

5. Tabulate the different value of $d V / d Q$.

6. Summation of all value of the above tabulated values will be the denominator in the formula used.

7. Each individual values in the above table will be the numerator in the formula.

8. Find out Pdiff (Shaft power-Electrical power).

9. Find out value of load to be shed using the classical formula.

$$
\left(\frac{d v i}{d q i}\right)
$$

S(load to be shed $)=$

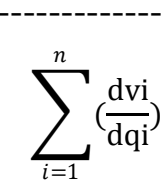

10. From the above values the tabulation will be made and it is shown in TABLE I below.

11. The above tabulation will be used in the formation of fuzzy expert system for the given power system network.

The following idea can be expressed as a simple flow chart.

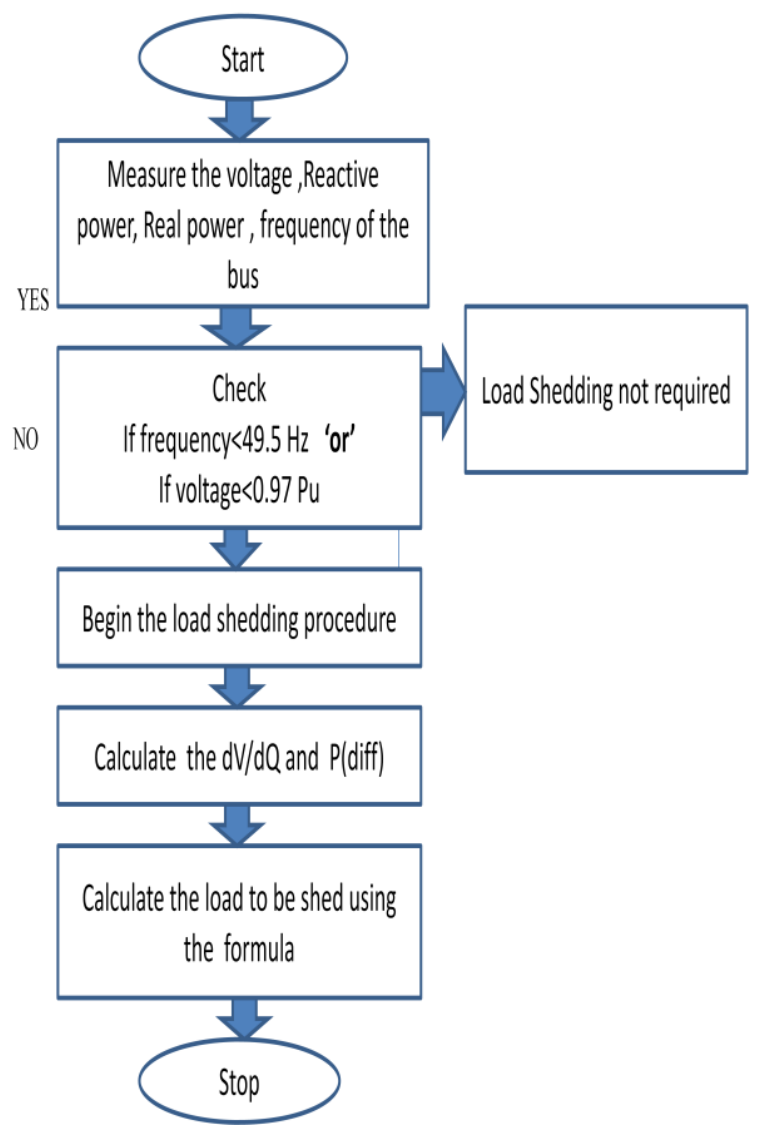

\section{FUZZY APPLICATION INMINIMISING THE LOAD SHEDDING}

Fuzzy control, which directly uses fuzzy rules, is the most important application in fuzzy theory. Using a procedure originated by Ebrahim Mamdani in the late 70s, three steps are taken to create a fuzzy controlled machine.

1) Fuzzification (Using membership functions to graphically describe a situation)

2) Rule evaluation (Application of fuzzy rules)

3) Defuzzification (Obtaining the crisp or actual results)

The following data's obtained from the substation and from the values of V, Q, and Pdiff by applying the classical formula the amount of load to be shed is calculated and given below. 
Table I

The Values of V, Q, Pdiff\& Load to Be Shed
LOAD TO BE SHED (MW)

0- 4.5-9-LOW (MF1)

9-13.5-18-MEDIUM (MF2)

18-22.5-27-HIGH (MF3)

\begin{tabular}{|c|c|c|c|}
\hline $\begin{array}{c}\text { VOLTAGE } \\
\text { (KV) }\end{array}$ & Q & Pdiff & $\begin{array}{c}\text { LOAD } \\
\text { TO }\end{array}$ \\
(MVAR) & (MW) & $\begin{array}{c}\text { BE SHED } \\
\text { (MW) }\end{array}$ \\
\hline 101 & 49 & 9 & 3.18 \\
\hline 101 & 49 & 18 & 18.59 \\
\hline 103 & 54 & 3 & 6.13 \\
\hline 103 & 29 & 33 & 14.09 \\
\hline 104 & 45 & 27 & 17.19 \\
\hline 104 & 50 & 24 & 17.19 \\
\hline 105 & 39 & 24 & 10.32 \\
\hline 105 & 39 & 17 & 10.59 \\
\hline 106 & 45 & 30 & 11.43 \\
\hline 106 & 30 & 39 & 28.19 \\
\hline 106 & 59 & 35 & 15 \\
\hline 107 & 40 & 39 & 21.20 \\
\hline 107 & 44 & 18 & 8.29 \\
\hline 107 & 54 & 3 & 2.64 \\
\hline 108 & 45 & 27 & 5.71 \\
\hline 109 & 34 & 27 & 0.448 \\
\hline
\end{tabular}

7. ASSIGNING MEMBERSHIP FUNCTION FOR INPUT \& OUTPUT VARIABLES

INPUTVOLTAGE (L1)

190-195-200-LOW (MF1)

200-205-210-MEDIUM (MF2)

210-215-220-HIGH (MF3)

\section{REACTIVE POWER (L2)}

25-31-38-LOW (MF1)

38-45-52-MEDIUM (MF2)

52-59-66-HIGH (MF3)

\section{Pdiff (L3)}

0-7-14-LOW (MF1)

14-21-28-MEDIUM (MF2)

28-35-42- HIGH (MF3)

\section{OUTPUT}

\section{FORMING FUZZY \\ LINGUISTICCONTOL TABLE}

Table II

Fuzzy Linguistic Control Table for 230 KV Bus System

\begin{tabular}{|l|l|c|c|}
\hline $\begin{array}{l}\text { Input 1 } \\
\text { voltage }\end{array}$ & $\begin{array}{c}\text { Input 2 } \\
\text { mivar }\end{array}$ & $\begin{array}{c}\text { Input 3 } \\
\text { mw }\end{array}$ & Output \\
\hline high & high & high & high \\
\hline high & high & medium & medium \\
\hline medium & medium & high & medium \\
\hline
\end{tabular}

Table III

Fuzzy Linguistic Control Table for 110 KV Bus System

\begin{tabular}{|c|c|c|c|}
\hline $\begin{array}{c}\text { Input 1 } \\
\text { voltage }\end{array}$ & $\begin{array}{c}\text { Input 2 } \\
\text { mvar }\end{array}$ & $\begin{array}{c}\text { Input 3 } \\
\text { mw }\end{array}$ & Output \\
\hline medium & low & low & low \\
\hline low & low & medium & medium \\
\hline
\end{tabular}

\section{FUZZY SCALING AND ITS CORRESPONDING OUPUT (LOAD TO BE SHED)}

Fuzzy linguistic rules are formed, for the input parameters and for the different conditions the fuzzy outputs are obtained and are as shown below. These fuzzy outputs are collectively tabulated below which is shown in TABLE IV and TABLE V.

TABLE IV shows the results for $110 \mathrm{KV}$ bus and TABLE V shows the results for $230 \mathrm{KV}$ bus. 

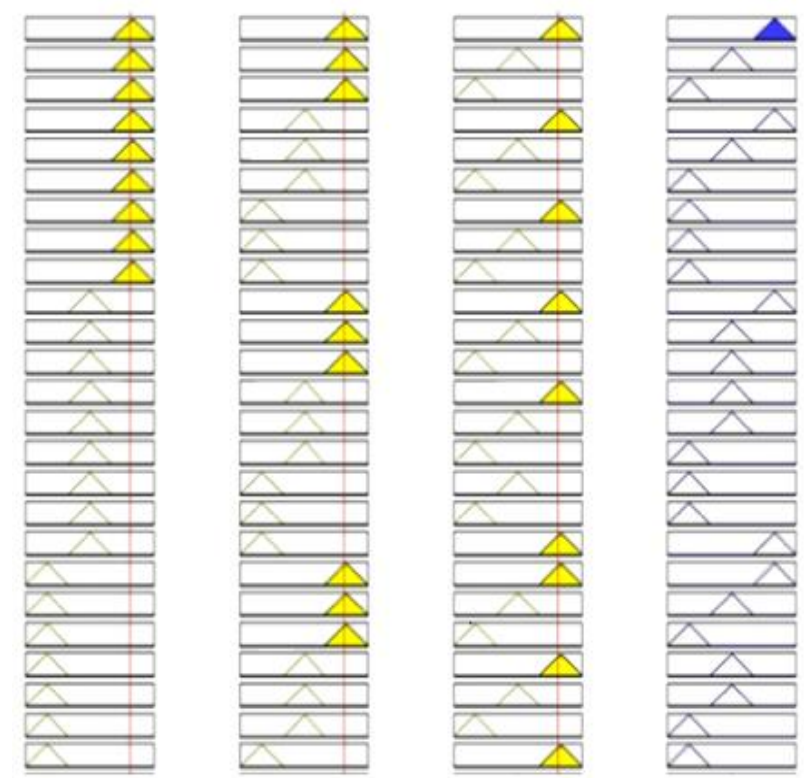

Figure 1: Condition 1: If Voltage is high, Reactive Power is high and Real Power is high then output is high.

Voltage $($ KV $)=216 \quad Q($ MVAR $)=62.1 \quad$ Pdiff $=23 \quad$ Load to be Shed=17
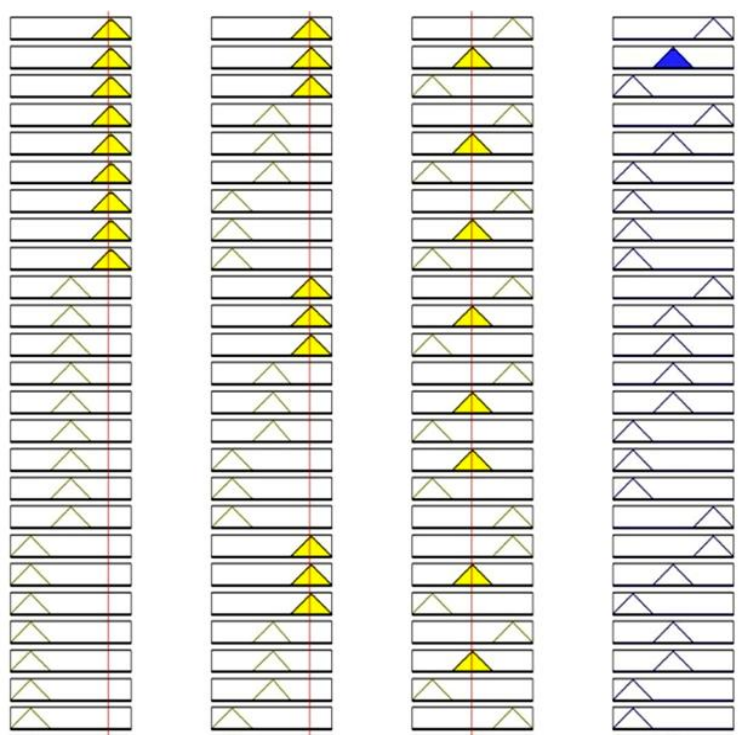

Figure 2: Condition 2: If Voltage is high, Reactive power is high and Real power is medium then output is medium.
Voltage $=210 \quad Q \quad($ MVAR $)=37 \quad$ Pdiff $=13 \quad$ Load to be shed $=8$
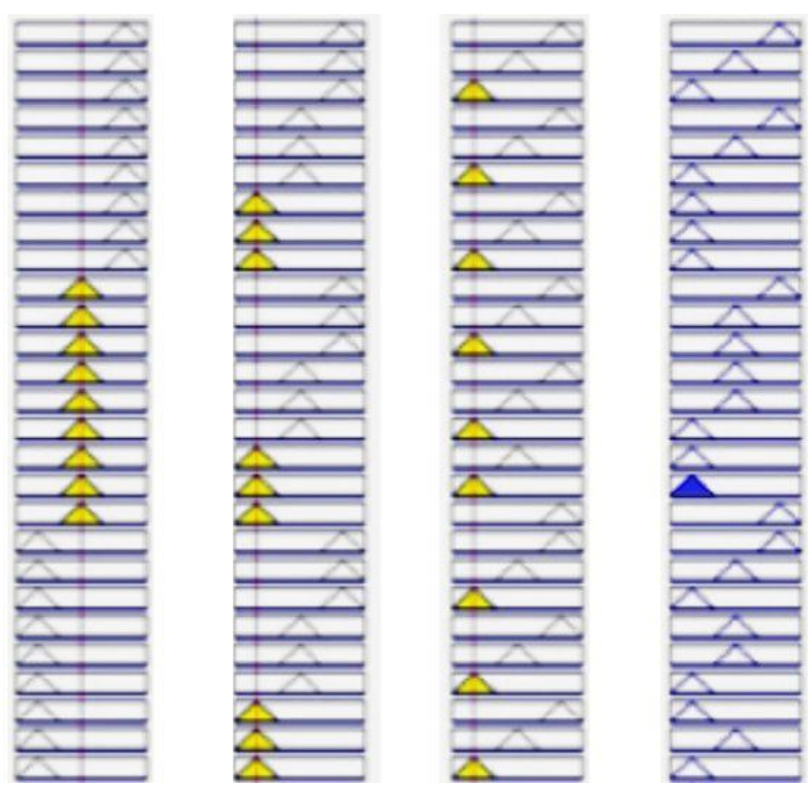

Figure 3: Condition 3: If Voltage is Medium, Reactive Power is Low, Real Power is Low then Output is Low.

Voltage $=195 \quad$ Q $($ MVAR $)=27 \quad$ Pdiff $=24 \quad$ Load to be Shed $=18$

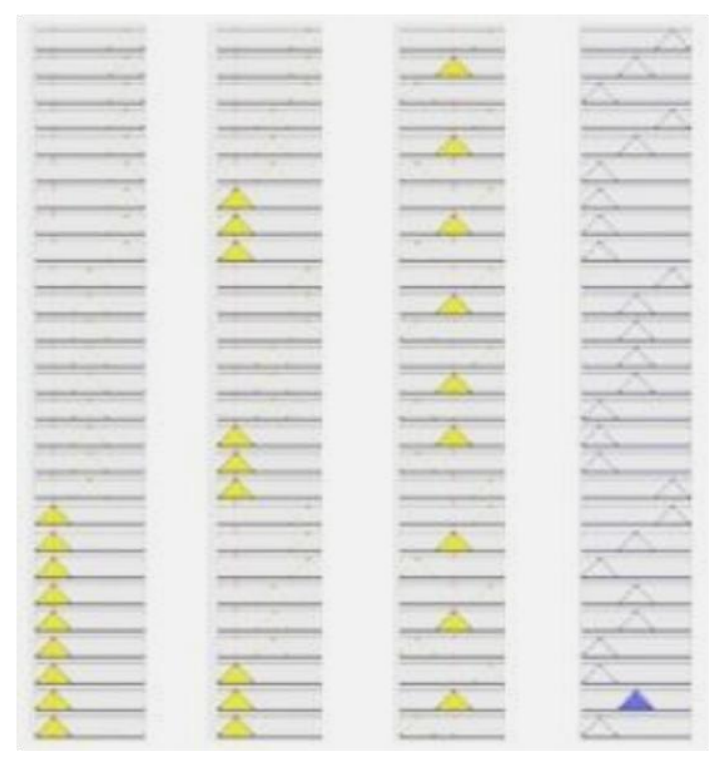

Figure 4: Condition 4: If Voltage is Low, Reactive Power is low and Real Power is medium then Output is medium. 


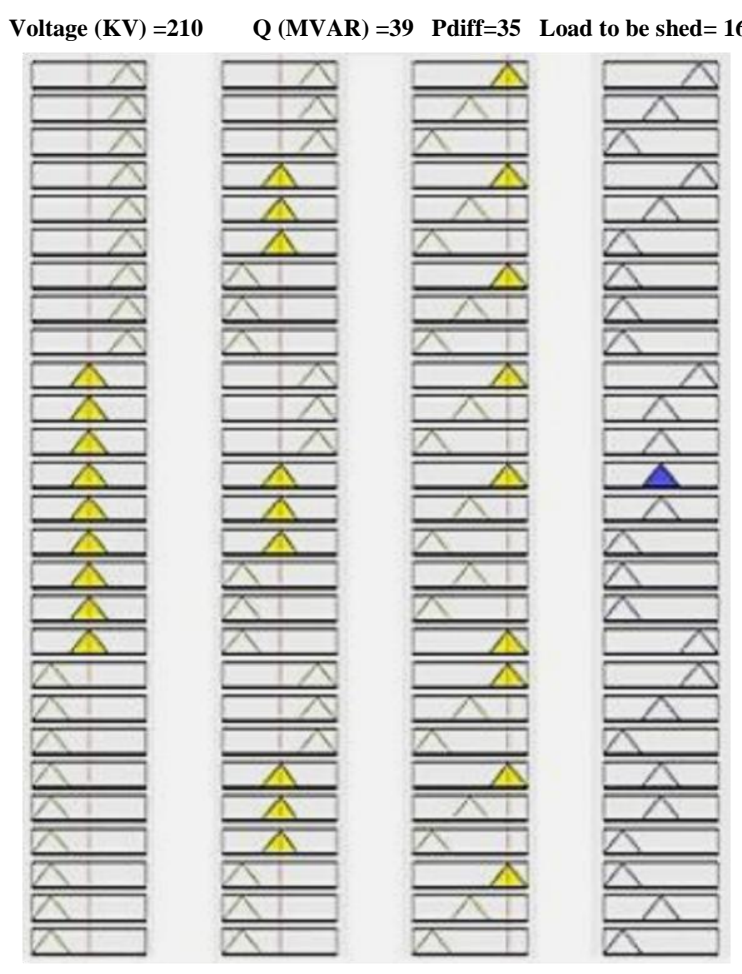

Figure 5: Condition 5: If Voltage is Medium, Reactive Power is medium and Real Power is high then output is medium.

\section{RESULTS \& DISCUSSIONS}

So far the data's of $110 \mathrm{kV}$ and $230 \mathrm{kV}$ bus systems are scaled using fuzzy expert system. From the data's obtained from the substation, this clearly indicates that there is a significant benefit over conventional methods.

Also taking three critical parameters say voltage, reactive power, shaft power or real power and applying these data in fuzzy expert system gives the output of the value of load to be shed, which is much less than the system without fuzzy.

This is applied for both $110 \mathrm{kv}$ and $230 \mathrm{kV}$ systems and the results reinforce the same idea. Here Mat lab fuzzy tool box is applied for data scaling and to minimize the load shedding.

With the above procedure an effective value of amount of load to be shed is arrived.

The results are tabulated below.
Table IV

\section{Comparative Results between Conventional Method \&} Fuzzy System Approach (For 110 KV Bus)

\begin{tabular}{|c|c|c|c|}
\hline $\begin{array}{c}\text { SL. } \\
\text { NO }\end{array}$ & $\begin{array}{c}\text { VOLTAGE } \\
(\mathrm{KV})\end{array}$ & $\begin{array}{c}\text { LOAD TO BE } \\
\text { SHED(CONVENTIONAL) } \\
\text { IN (MW) }\end{array}$ & $\begin{array}{c}\text { LOAD TO BE } \\
\text { SHED(FUZZIFIED) } \\
\text { IN (MW) }\end{array}$ \\
\hline 1. & 101 & 13.83 & 3.18 \\
\hline 2. & 103 & 7.44 & 6.13 \\
\hline 3. & 104 & 20.12 & 17.19 \\
\hline 4. & 105 & 24.12 & 10.59 \\
\hline 5. & 106 & 22.1 & 15 \\
\hline 6. & 107 & 24.18 & 21.20 \\
\hline 7. & 108 & 26.17 & 5.71 \\
\hline 8. & 109 & 15.32 & 4.48 \\
\hline
\end{tabular}

\section{Table V}

Comparative Results between Conventional Method \& Fuzzy System Approach (For 230 KV Bus)

\begin{tabular}{|c|c|c|c|}
\hline $\begin{array}{c}\text { SL. } \\
\text { NO }\end{array}$ & $\begin{array}{c}\text { VOLTAGE } \\
(\mathrm{KV})\end{array}$ & $\begin{array}{c}\text { LOAD TO BE } \\
\text { SHED(CONVENTIO } \\
\text { NAL) IN (MW) }\end{array}$ & $\begin{array}{c}\text { LOAD TO BE } \\
\text { SHED(FUZZIFIED) } \\
\text { IN (MW) }\end{array}$ \\
\hline 1. & 199 & 14.72 & 0.705 \\
\hline 2. & 203 & 13.83 & 15.8 \\
\hline 3. & 205 & 20.12 & 16.6 \\
\hline 4. & 209 & 20.88 & 14 \\
\hline 5. & 211 & 24.18 & 11.3 \\
\hline 6. & 213 & 13.32 & 18 \\
\hline 7. & 215 & 26.37 & 15.8 \\
\hline 8. & 217 & 16.2 & \\
\hline
\end{tabular}

The above results are shown graphically below where the yellow line indicates the system without fuzzy and green line indicates system with fuzzy. Two separate graphical displays are shown each for $110 \mathrm{KV}$ bus and $230 \mathrm{KV}$. 


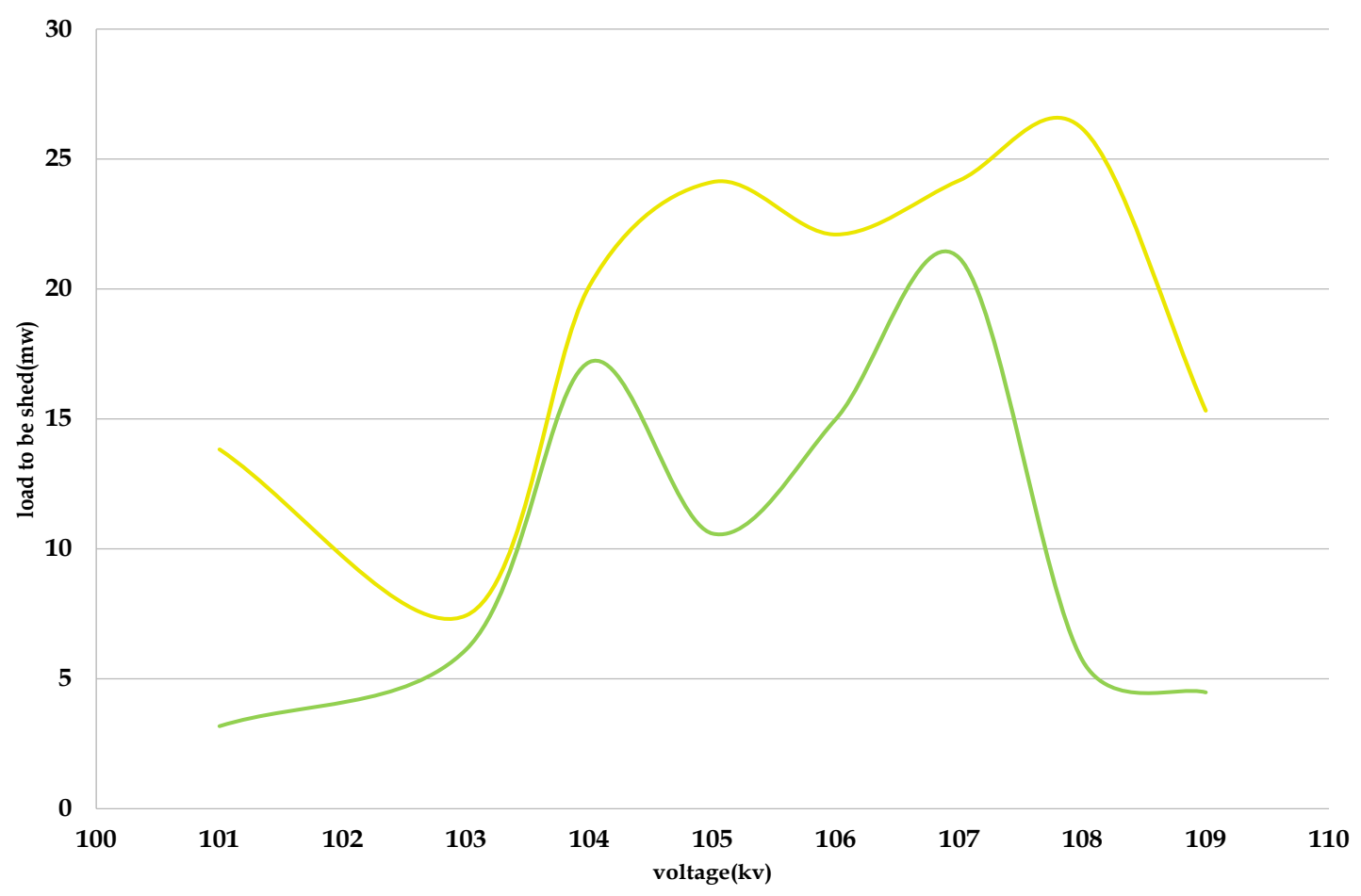

Figure 6: Graph That Represents The Comparison Between The Conventional Method And Fuzzy Expert Systems, Output Value Of Amount Of Load To Be Shed For 110kv Bus System.

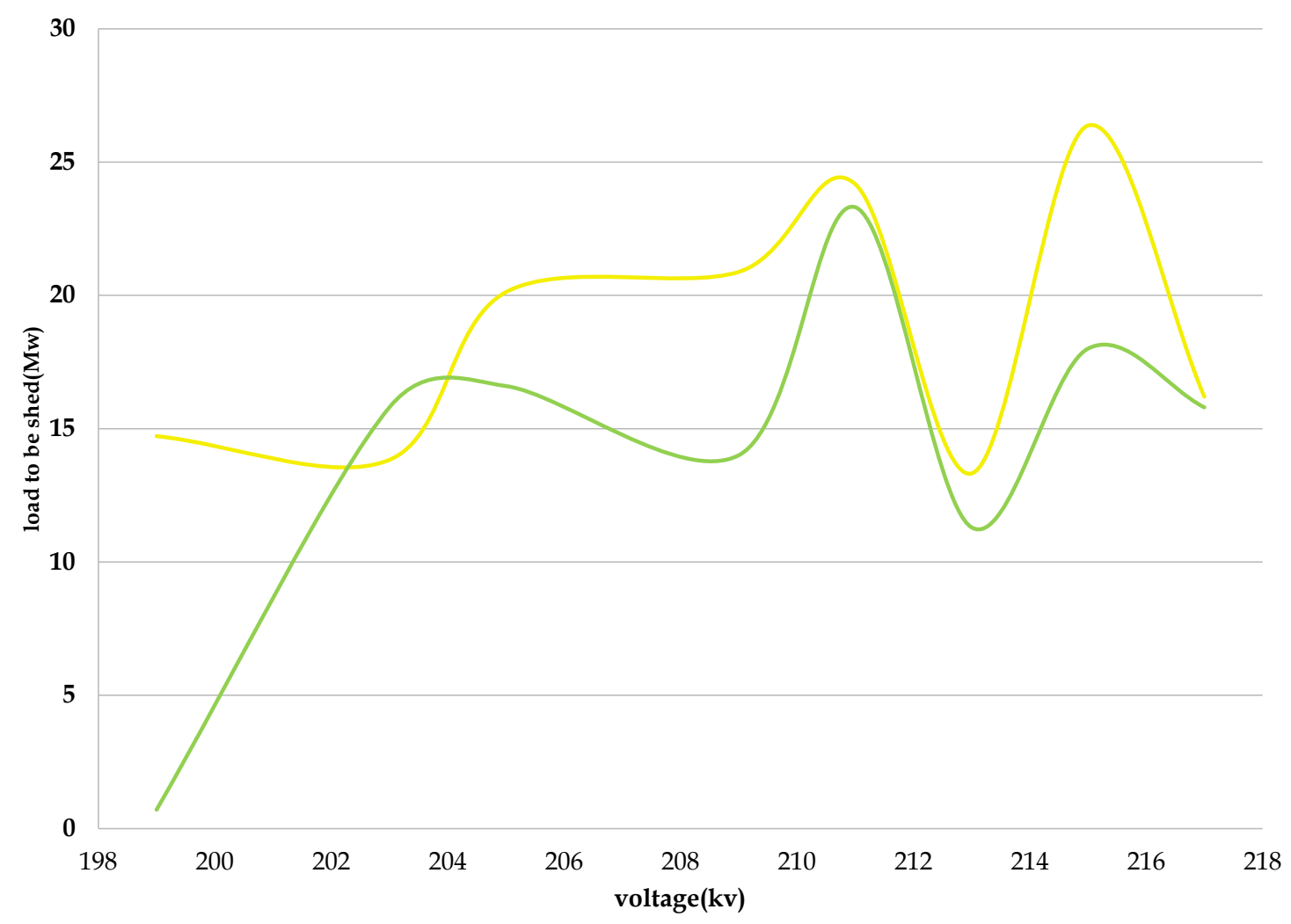

Figure7: Graph that represents the comparison between the conventional method and fuzzy expert systems, output value of amount of load to be shed for $230 \mathrm{kv}$ bus system. 


\section{CONCLUSIONS}

From the table numbers IV and V it is clear that fuzzy scaling of critical parameters and writing the fuzzy linguistic rules properly, has clearly given good results. The amount of load to be shed is minimized. This method or this work has been developed from the data's obtained from the substation and the discussions with the field engineers. It is a logical thought by some ways and means the load shedding has to be minimized. Even though load shedding is the last resort and practically followed method to relieve congestion, there is a constant urge both from field engineers and academic circle to analyze and find some ways to minimize the load shedding. In that regard this work can be respected. By building robust electronics in such a way that the fuzzy outputs are tuned to a fuzzy controller which is again governed by another control device with feedback system and data acquisition system to monitor the bus parameters, when this forms a robust loop, this idea can be tested and if the results are satisfactory this can be implemented in distribution substations.

\section{REFERENCES}

[1] Seyed Abbas Taher, Hadi Besharat., "Transmission Congestion Management by determining Optimal Location of FACTS Devices in Deregulated Power Systems", American Journal of Applied Sciences Publications, 2008.

[2]. Kaiumuzzaman Mollah, Nirmal C-K Nair, "Coordinated Strategy for Under-voltage and Under-frequency Load Shedding", IEEE Transactions.
[3]. T.Quoc Tuan, J.Fandino, N.Hadjsaid, J.C. Sabonnadi and $\mathrm{H} . \mathrm{Vu}$, Emergency Load Shedding to Avoid Risk of Voltage Instability Using Indicators, IEEE Transactions on Power Systems, Vol.9,No. 1 February 1994.

[4]. Vladimir V. Terzijia, "Adaptive Under frequency Load Shedding Based on the Magnitude of the Disturbance Estimation", IEEE Transactions on Power Systems, Vol.21, 2006.

[5]. Y.Wang, I.R.Pordanjani, W.Li, E.Vaahedi, "Strategy to Minimize the load shedding amount for voltage collapse prevention", IET Gener.Transm. Distrib, 2011, Vol.5, Iss.3, pp.307-313.

[6]. Ragu Balanathan, Nalin C. Pahalawaththa, Udaya D. Annakkage, "A Strategy for Under voltage Load shedding in Power Systems" 0-7803-4754-4/98/\$10.00 01998 IEEE.

[7]. A.M. Leite da Silva ' A.M. Cassula ' R. Billinton L.A.F. Manso "Optimum Load Shedding Strategies in Distribution Systems" Power Tech '01 Conference, Porto, Portugal, August 29 -September 2, 2001, IEEE.

[8]. Charles Mozin, Under-voltage load shedding, Consultant, Beckwith Electric Co. Inc.

[9] L.-X Wang, "A Course in Fuzzy Systems and Control", Englewood Cliff, NJ: Prentice-Hall PTR, 1997, ch. 2, 4, and 29 . 